\title{
One-dimensional solidification of supercooled melts
}

\author{
F. Font ${ }^{1,2}$, S. L. Mitchell ${ }^{3}$, and T. G. Myers ${ }^{1}$ \\ ${ }^{1}$ Centre de Recerca Matemàtica, Campus de Bellaterra, Edifici C, 08193 Bellaterra, Barcelona, Spain \\ ${ }^{2}$ Departament de Matemàtica Aplicada I, Universitat Politècnica de Catalunya, Barcelona, Spain \\ ${ }^{3}$ MACSI, Department of Mathematics and Statistics, University of Limerick, Limerick, Ireland
}

December 4, 2012

\begin{abstract}
In this paper a one-phase supercooled Stefan problem, with a nonlinear relation between the phase change temperature and front velocity, is analysed. The model with the standard linear approximation, valid for small supercooling, is first examined asymptotically. The nonlinear case is more difficult to analyse and only two simple asymptotic results are found. Then, we apply an accurate heat balance integral method to make further progress. Finally, we compare the results found against numerical solutions. The results show that for large supercooling the linear model may be highly inaccurate and even qualitatively incorrect. Similarly as the Stefan number $\beta \rightarrow 1^{+}$the classic Neumann solution which exists down to $\beta=1$ is far from the linear and nonlinear supercooled solutions and can significantly overpredict the solidification rate.

Keywords: Phase change; Stefan problem; kinetic undercooling; supercooling; heat balance integral method; asymptotic solutions; similarity solutions
\end{abstract}

\section{Introduction}

Supercooled liquids can solidify much more rapidly than a non-supercooled liquid and when rapid solidification occurs the liquid may not have time to form its usual crystalline structure. Materials made from supercooled melts can therefore have markedly different properties to the standard form of the material. A material formed from a supercooled liquid, usually called a glassy or amorphous solid, can present greater corrosion resistance, toughness, strength, hardness and elasticity than common materials: amorphous metal alloys can be twice as strong and three times more elastic than steel. Such materials are currently used in medicine, defence and aerospace equipment, electronics and sports $[1,2,3]$. Recent advances in the production and use of amorphous solids provides the motivation for this theoretical study on the solidification of a supercooled liquid.

Theoretical investigations of Stefan problems have focussed primarily on the situation where the phase change temperature is constant. However, there are various applications where this temperature changes from its standard value (the heterogenoeus nucleation temperature) and may even vary with time. One method to reduce the freezing point is to increase the ambient pressure. This method is exploited in the food industry, whereby the sample is cooled well below the normal freezing temperature by applying a high pressure. The pressure is then released and almost instantaneous freezing occurs. This permits the freezing of certain products that normally spoil when frozen more slowly. The technique is also used in cryopreservation [4]. Pressure may also vary due to surface tension effects at a curved interface. Hence freezing fronts with high curvature may exhibit 
a variable phase change temperature. Another mechanism for varying the phase change temperature occurs when a liquid is supercooled or undercooled (we will use both terms in the following work), that is, the liquid is cooled below the heterogeneous nucleation temperature. In this situation the liquid molecules have little energy which affects their mobility and hence their ability to move to the solid interface $[5,6]$.

In this paper we focus on the final mechanism, where the liquid is supercooled. In the standard Stefan problem the phase change temperature is specified as a constant, say $T_{m}$, and the speed of the phase change front is related to the temperature gradient in the surrounding phases. When modelling the solidification of a supercooled liquid, the phase change temperature is unknown and so a further equation is required, which relates the speed of the front to the degree of supercooling. If we denote the temperature at which the phase change occurs as $T_{I}$ and $s(t)$ as the position of the front then a typical form for the relation between $s_{t}$ and the degree of supercooling is shown in Figure 1. The left hand plot represents the copper solidification process, the right hand plot represents salol (which occurs at a slow rate and so provides relatively simple experiments). Both curves have the same qualitative form. For a small degree of supercooling, i.e. for copper $T_{m}-T_{I}<250$ $\mathrm{K}$, the speed of the front increases as the supercooling increases. This behaviour seems physically sensible, the cooler the sample the more rapid the freezing. However, for larger supercooling the process slows down as the molecules become more 'sluggish' due to a lack of energy. The maximum solidification rate for copper is around $2.9 \mathrm{~m} / \mathrm{s}$, for salol it is around $4.4 \times 10^{-5} \mathrm{~m} / \mathrm{s}$ (making salol a more popular choice for experimental studies).
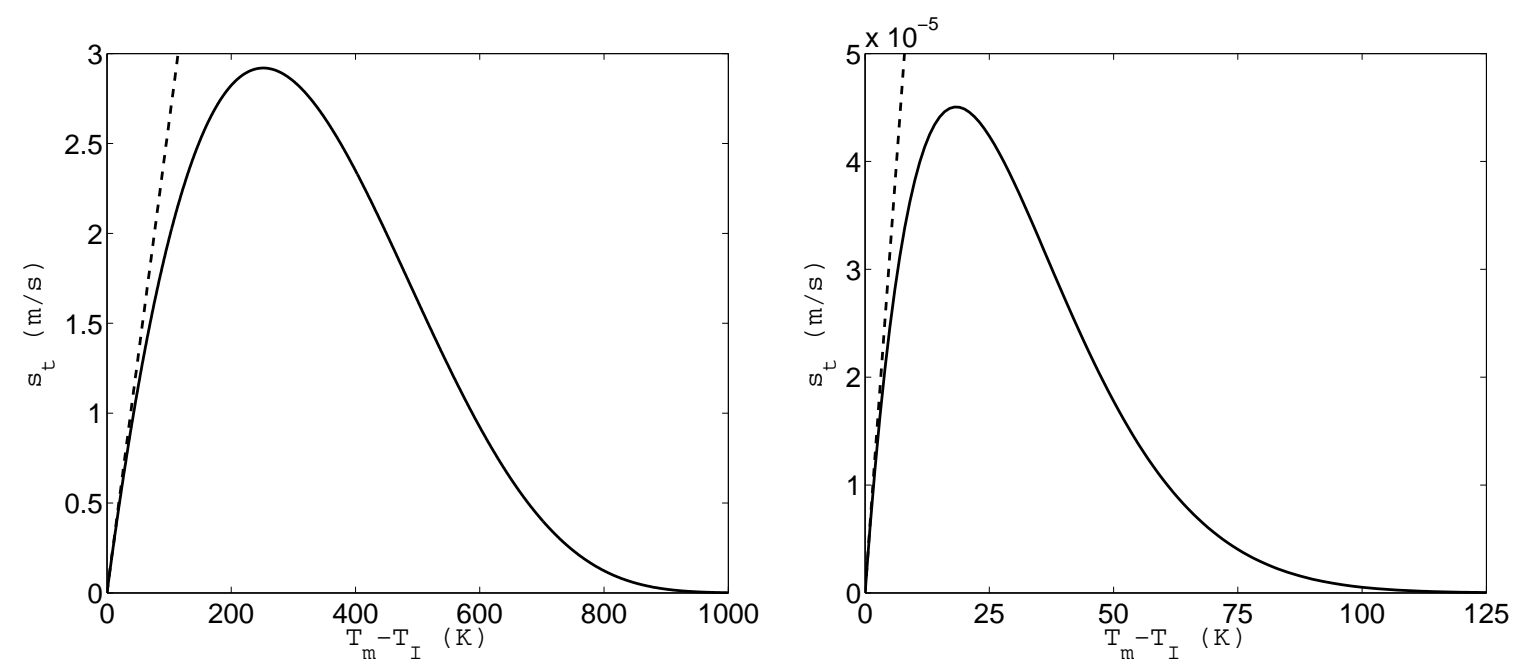

Figure 1: Representation of the solidification speed of copper (left) and salol (right) as a function of the supercooling. The solid line represents the full expression for $s_{t}$, the dashed line the linear approximation.

Using a statistical mechanics argument, it is shown in Ashby \& Jones [5, Chap. 6] that the solidification rate may be approximated by

$$
s_{t}=\frac{d \Delta h}{6 \hbar T_{m}} e^{-\frac{q}{k T_{I}}}\left(T_{m}-T_{I}\right),
$$

where $d$ is the molecular diameter, $\hbar$ the Planck constant, $q$ the activation energy and $k$ the Boltzmann constant. The parameter $\Delta h$ is the latent heat per particle, calculated by dividing the latent heat of fusion $\left(L_{m}\right)$ by Avogadro's number $\left(N_{A}\right)$. A linearised version of equation (1) is often dealt with in the literature, $[7,8,9,10,11]$,

$$
T_{I}(t)=T_{m}-\phi s_{t},
$$

where $\phi=6 \hbar T_{m} e^{\frac{q}{k T_{m}}} /(d \Delta h)$. This expression for $\phi$ provides one interpretation of the usual kinetic undercooling coefficient described, for example, in $[6,7,9]$. The solid lines 
in Figure 1 were obtained by plotting equation (1) using the parameter values of Table 1 , the broken lines come from equation (2).

The classical Neumann solution to the Stefan problem with fixed boundary and constant far field temperature requires setting $\phi=0$ in (2). With the Neumann solution the interface velocity increases as the Stefan number $\beta$ decreases: as $\beta \rightarrow 1^{+}$the velocity tends to infinity and the Neumann solution breaks down. In order to obtain solutions for Stefan numbers $\beta \leq 1$, numerous authors have adopted the linear profile, with $\phi \neq 0$, which removes the infinite boundary velocity. Incorporating the effects of the linear interfacial kinetics into the Stefan problem, by using (2), results in different solution behaviour depending on the value of $\beta$ : for $\beta>1$ the velocity $s_{t} \propto t^{-1 / 2}$ (as occurs with the Neumann solution), when $\beta=1$ it changes to $s_{t} \propto t^{-1 / 3}$ and for $\beta<1, s_{t}$ is constant (and the temperature is a travelling wave solution), see [6]. The short time solution given in [7] has $s_{t}$ constant at leading order with a first order correction of $t^{1 / 2}$, valid for all $\beta$.

In the following work we will study the one-phase solidification process subject to arbitrary supercooling and so employ equation (1) to determine the interface temperature $T_{I}$. In $\S 3.2$ we employ an asymptotic analysis to the linear supercooled model to reproduce all the different behaviours mentioned above. In $\S 3.3$ we find a small time solution, valid for all $\beta$, and a large time solution valid for $\beta<1$. In $\S 4$ we describe an accurate Heat Balance Integral Method (HBIM) and show how the resultant equations can reproduce all the behaviours predicted for linear supercooling. Further, using this method we can find the same range of behaviour for nonlinear supercooling at large times. In $\S 5$ we present results for the asymptotic and HBIM models and compare them with a numerical solution.

\section{Mathematical Model}

We consider a one-dimensional supercooled liquid initially occupying $x \geq 0$, with solidification starting at the point $(x, t)=(0,0)$. An appropriate one-phase Stefan problem is then specified by

$$
\begin{array}{rlrl}
\frac{\partial T}{\partial t} & =\alpha_{l} \frac{\partial^{2} T}{\partial x^{2}}, & & s<x<\infty \\
T & =T_{I}(t), \quad \rho_{l} L_{m} s_{t}=-k_{l} \frac{\partial T}{\partial x}, \quad \text { at } x=s \\
T & \rightarrow T_{\infty}, \quad \text { as } x \rightarrow \infty, \\
T(x, 0) & =T_{\infty}, \quad s(0)=0, &
\end{array}
$$

where $T_{\infty}<T_{m}$. The parameters $\alpha_{l}, \rho_{l}, L_{m}, k_{l}$ represent the thermal diffusivity, density, latent heat and conductivity respectively. Typical parameter values are presented in Table 1. The interface temperature is related to the interface velocity by equation (1). The linearised version is given by (2) and setting $\phi=0$ in this equation brings us back to the standard condition that the interface temperature takes the constant value $T_{m}$. Note, the system (3)-(6) in fact loses energy. In [9] this issue is discussed in detail and an energy conserving form is presented which is valid in the limit $k_{s} / k_{l} \rightarrow 0$. However, this limit is physically unrealistic and in [12] the reduction in the limit $k_{s} / k_{l} \rightarrow \infty$ is determined. Results there are shown for the two limiting cases, the basic reduction of (3)-(6) and numerics for the full two-phase problem. It is shown that the reduction (3)-(6) gives a reasonable approximation and is in fact more accurate than the energy conserving form with $k_{s} / k_{l} \rightarrow 0$. So, since (3)-(6) is the most common form we retain it for this paper.

The system (3)-(6) may be nondimensionalised with

$$
\hat{T}=\frac{T-T_{m}}{\Delta T}, \quad \hat{t}=\frac{t}{\mathcal{T}}, \quad \hat{x}=\frac{x}{L}, \quad \hat{s}=\frac{s}{L}, \quad \hat{T}_{I}=\frac{T_{I}-T_{m}}{\Delta T},
$$

where $\mathcal{T}=L^{2} / \alpha_{l}$ and $L=\phi \alpha_{l} / \Delta T$. The temperature scale is $\Delta T=T_{m}-T_{\infty}$. The lengthscale is determined from equation (1), however if $T_{I}=T_{m}$ there is no natural length-scale 


\begin{tabular}{|c||c|c|c|c|c|c|c|}
\hline \hline Substance & $\begin{array}{c}T_{m}(\mathrm{~K}) \\
\left(\times 10^{3}\right)\end{array}$ & $\begin{array}{c}L_{m}(\mathrm{~J} / \mathrm{Kg}) \\
\left(\times 10^{5}\right)\end{array}$ & $\begin{array}{c}c_{l}(\mathrm{~J} / \mathrm{Kg} \cdot \mathrm{K}) \\
\left(\times 10^{3}\right)\end{array}$ & $\begin{array}{c}\rho_{l}\left(\mathrm{~kg} / \mathrm{m}^{3}\right) \\
\left(\times 10^{3}\right)\end{array}$ & $k_{l}(\mathrm{~W} / \mathrm{m} \cdot \mathrm{K})$ & $\begin{array}{c}q(\mathrm{~J}) \\
\left(\times 10^{-20}\right)\end{array}$ & $\begin{array}{c}d(\mathrm{~m}) \\
\left(\times 10^{-9}\right)\end{array}$ \\
\hline \hline Copper & 1.36 & 2.09 & 0.53 & 8.02 & 157.18 & 6.71 & 0.23 \\
\hline Salol & 0.32 & 0.90 & 1.58 & 1.18 & 0.18 & 6.61 & 1.00 \\
\hline \hline
\end{tabular}

Table 1: Approximate thermodynamical parameter values for $\mathrm{Cu}$ and Salol, taken from $[5,13,14,15]$.

and it must be left arbitrary. Dropping the hat notation the governing equations are now

$$
\begin{array}{rlrlrl}
\frac{\partial T}{\partial t} & =\frac{\partial^{2} T}{\partial x^{2}}, & & s<x<\infty & & \\
T & =T_{I}(t), & & \beta s_{t}=-\frac{\partial T}{\partial x}, & & \text { at } x=s \\
T & \rightarrow-1, & & \text { as } x \rightarrow \infty \\
T(x, 0) & =-1, & & s(0)=0,
\end{array}
$$

where $\beta=L_{m} /\left(c_{l} \Delta T\right)$. The nondimensional interface temperature $T_{I}$ is determined from

$$
s_{t}=-T_{I} \exp \left(\frac{Q T_{I}}{P+T_{I}}\right)
$$

where $Q=q /\left(k T_{m}\right)$ and $P=T_{m} / \Delta T$. The case of no supercooling is retrieved by setting $T_{I}(t)=0$ in (9) and then (12) is not used, small supercooling requires $\Delta T$ to be small such that $P \rightarrow \infty$ and the exponential term of (12) may be set to unity, then $s_{t}=-T_{I}(t)$. Otherwise, the full expression in (12) is used to determine $T_{I}$.

When $T_{I}(t)$ is not identically zero then we require an initial value (and also one for $\left.s_{t}\right)$. Noting that $T(x, 0)=-1$ and $T_{I}(t)=T(s, t)$ then $s(0)=0$ indicates that $T_{I}(0)=$ $T(0,0)=-1$. An initial (finite) value for $s_{t}$ may then be found by substituting $T_{I}(0)=-1$ into equation (12). The linearised version simply leads to $s_{t}(0)=1$. In the case $T_{I} \equiv 0$ then as $t \rightarrow 0$ the discontinuity in temperature due to the conflicting conditions $T(0,0)=$ $0, T(x, 0)=-1$ cannot be avoided. This indicates that the front velocity $s_{t}=-T_{x}(s, t)$ is infinite at $t=0$ (this will become clear in subsequent sections).

Before proceeding further, it is convenient to immobilise the boundary by setting

$$
y=x-s, \quad T(x, t)=F(y, t) .
$$

Then the system (8)-(11) becomes

$$
\begin{aligned}
\frac{\partial^{2} F}{\partial y^{2}} & =\frac{\partial F}{\partial t}-s_{t} \frac{\partial F}{\partial y}, \quad 0<y<\infty \\
F & =T_{I}(t), \quad \beta s_{t}=-\frac{\partial F}{\partial y}, \quad \text { at } y=0 \\
F & \rightarrow-1, \quad \text { as } y \rightarrow \infty \\
F(y, 0) & =-1, \quad s(0)=0 .
\end{aligned}
$$

In the following we will analyse this system further.

\section{Asymptotic analysis}

Most exact solutions to Stefan problems, where they exist, can be obtained by similarity methods. Hence, we begin by looking for similarity solutions to the system (14)-(17) and so define

$$
\eta=\frac{y}{t^{\alpha}}, \quad F(y, t)=G(\eta) .
$$


The governing equations (14)-(16) are now

$$
\begin{aligned}
G^{\prime \prime} & =-\left(\alpha \eta t^{2 \alpha-1}+t^{\alpha} s_{t}\right) G^{\prime}, \quad 0<\eta<\infty \\
G(0) & =T_{I}(t),\left.\quad G\right|_{\eta \rightarrow \infty} \rightarrow-1, \quad \beta t^{\alpha} s_{t}=-G^{\prime}(0),
\end{aligned}
$$

with $s(0)=0$, and primes denote differentiation with respect to $\eta$. Denoting $\nu=$ $-G^{\prime}(0) / \beta \geq 0$ we use the Stefan condition to eliminate $t^{\alpha} s_{t}$ from (19) and so the model becomes

$$
\begin{aligned}
G^{\prime \prime} & =-\left(\alpha \eta t^{2 \alpha-1}+\nu\right) G^{\prime}, \quad 0<\eta<\infty \\
G(0) & =T_{I}(t),\left.\quad G\right|_{\eta \rightarrow \infty} \rightarrow-1, \quad t^{\alpha} s_{t}=\nu, \quad s(0)=0 .
\end{aligned}
$$

Since $G=G(\eta)$ this system is strictly only valid when the $t$ dependence is removed from the equations involving $G$. In (21) this is achieved by setting $\alpha=0$ or $1 / 2$ but then we must also take into account the boundary condition $G(0)=T_{I}(t)$.

\subsection{No kinetic undercooling}

The classical formulation for the one-phase supercooled Stefan problem with no kinetic undercooling is obtained by setting $T_{I}(t)=0$. In this case we choose $\alpha=1 / 2$ and so the governing equations (21)-(22) are now

$$
\begin{array}{rlrl}
G^{\prime \prime} & =-\left(\frac{\eta}{2}+\nu\right) G^{\prime}, & 0<\eta<\infty \\
G(0) & =0, \quad t^{1 / 2} s_{t}=\nu,\left.\quad G\right|_{\eta \rightarrow \infty} \rightarrow-1 .
\end{array}
$$

Integrating the Stefan condition gives $s=2 \nu \sqrt{t}$ and the solution for $G$ is

$$
G(\eta)=-1+\frac{\operatorname{erfc}(\eta / 2+\nu))}{\operatorname{erfc}(\nu)}, \quad s=2 \nu \sqrt{t} .
$$

The definition of $\nu=-G^{\prime}(0) / \beta$ then leads to the transcendental equation

$$
\beta \sqrt{\pi} \nu \operatorname{erfc}(\nu) e^{\nu^{2}}=1 .
$$

In the original variables the solution is

$$
T=-1+\frac{\operatorname{erfc}(x /(2 \sqrt{t}))}{\operatorname{erfc}(\nu)}, \quad s=2 \nu \sqrt{t}, \quad s_{t}=\frac{\nu}{\sqrt{t}},
$$

together with (26) this defines the well-known Neumann solution. The values of $\nu$ and hence the velocity of the phase change front are therefore dependent on $\beta=L_{m} /\left(c_{l} \Delta T\right)$. As mentioned earlier, this solution has infinite velocity at $t=0$ (since $s_{t} \propto 1 / \sqrt{t}$ ). Equation (26) determines $\nu(\beta)$, it has a unique solution for $\beta>1$ but when $\beta \leq 1$ no solution exists; as $\beta \rightarrow 1^{+}, \nu \rightarrow \infty$ and the front velocity is $\infty$ for all time.

\subsection{Linear kinetic undercooling}

\subsubsection{Small time solutions}

As discussed in Section $\S 2$, without kinetic undercooling the initial boundary temperature is fixed, $T(0,0)=0$, thus forcing an initial infinite temperature gradient. With kinetic undercooling $T(0,0)=T_{I}(0) \approx-1$ permits a finite temperature gradient. This indicates the need for different scalings at small times.

Let us now consider the governing equations (14)-(17). Noting that $F(y, 0)=-1$ we look for a small time similarity solution close to $F=-1$ and so re-define the function $G(\eta)$ as

$$
\eta=\frac{y}{t^{1 / 2}}, \quad F(y, t)=-1+t^{1 / 2} G(\eta) .
$$


This transforms equations (14)-(17) into

$$
\begin{aligned}
G^{\prime \prime} & =\frac{1}{2} G-\frac{\eta}{2} G^{\prime}-t^{1 / 2} s_{t} G^{\prime}, & & 0<\eta<\infty \\
-1+t^{1 / 2} G(0) & =-s_{t}, \quad \beta s_{t}=-G^{\prime}(0), & & \left.G\right|_{\eta \rightarrow \infty} \rightarrow 0 .
\end{aligned}
$$

The factor $t^{1 / 2}$ in front of $G(\eta)$ in (28) is chosen to balance the Stefan condition in (30) and then it follows that $s \propto t$. This leaves time in the governing equation (29) and the boundary condition (30a): for this reason we make the small time substitution $t=\epsilon \tau$, $s=\epsilon r$, where $\epsilon$ is an artificial small parameter [16, 17], to find

$$
\begin{aligned}
G^{\prime \prime} & =\frac{1}{2} G-\frac{\eta}{2} G^{\prime}-\epsilon^{1 / 2} \tau^{1 / 2} r_{\tau} G^{\prime}, \quad 0<\eta<\infty \\
-1+\epsilon^{1 / 2} \tau^{1 / 2} G(0) & =-r_{\tau}, \quad r_{\tau}=\nu,\left.\quad G\right|_{\eta \rightarrow \infty} \rightarrow 0 .
\end{aligned}
$$

This form motivates a perturbation series with $G(\eta)=G_{0}(\eta)+\mathcal{O}\left(\epsilon^{1 / 2}\right)$ and $r(\tau)=$ $r_{0}(\tau)+\epsilon^{1 / 2} r_{1}(\tau)+\mathcal{O}(\epsilon)$, i.e. the leading order form for $G$ is independent of $\tau$ but this is not necessarily true for $r$. The leading order problem is then

$$
\begin{aligned}
G_{0}^{\prime \prime} & =\frac{1}{2} G_{0}-\frac{\eta}{2} G_{0}^{\prime}, \quad 0<\eta<\infty \\
1 & =r_{0 \tau}, \quad r_{0 \tau}=\nu,\left.\quad G_{0}\right|_{\eta \rightarrow \infty}=0 .
\end{aligned}
$$

Combining the first two boundary conditions in (34) shows that $\nu=1$ and this provides the second boundary condition for $G_{0}$, namely $G_{0}^{\prime}(0)=-\beta\left(\right.$ since $\left.\nu=-G^{\prime}(0) / \beta\right)$. The solution is then

$$
G_{0}(\eta)=\beta\left[\frac{2}{\sqrt{\pi}} e^{-\eta^{2} / 4}-\eta \operatorname{erfc}\left(\frac{\eta}{2}\right)\right], \quad r_{0}(\tau)=\tau .
$$

Although the self-similarity assumption in (28) does not permit us to solve for the temperature at first order, equation (32a) indicates that

$$
r_{1 \tau}=-\tau^{1 / 2} G_{0}(0) \quad \Rightarrow \quad r_{1}=-\frac{4 \beta \tau^{3 / 2}}{3 \sqrt{\pi}},
$$

where we have used the value $G_{0}(0)=2 \beta / \sqrt{\pi}$. Writing this solution back in the original variables gives

$$
\begin{aligned}
T(x, t) & \approx-1+\beta\left[2 \sqrt{\frac{t}{\pi}} \exp \left(-\frac{(x-s)^{2}}{4 t}\right)-(x-s) \operatorname{erfc}\left(\frac{x-s}{2 \sqrt{t}}\right)\right], \\
s & \approx t\left[1-\frac{4 \beta}{3 \sqrt{\pi}} \sqrt{t}\right], \quad s_{t} \approx 1-\frac{2 \beta}{\sqrt{\pi}} \sqrt{t} .
\end{aligned}
$$

Recall that the temperature is accurate only to leading order whilst the position of the front is accurate to first order. Note, we will see the condition $s_{t} \rightarrow 1$ as $t \rightarrow 0$ arising below for various other cases.

\subsubsection{Large time solutions}

For sufficiently small times, the solution of the previous section holds for any value of $\beta$. The large time solution, on the other hand, takes different forms depending on whether $\beta$ is greater, less than or equal to 1 . 
Case $\beta<1$ : In this case we choose $\alpha=0$, in (18), and the governing equations (21)-(22) reduce to

$$
\begin{aligned}
G^{\prime \prime} & =-\nu G^{\prime}, \quad 0<\eta<\infty \\
G(0) & =-s_{t},\left.\quad G\right|_{\eta \rightarrow \infty} \rightarrow-1, \quad s_{t}=\nu, \quad s(0)=0 .
\end{aligned}
$$

Note, now $\eta=y=x-s$, which indicates a travelling wave solution. The travelling wave formulation differs from the other forms of similarity solution. In general the similarity transformation $\eta=y / t^{\alpha}$ with $\alpha \neq 0$ allows the far-field and initial conditions to be combined, that is $\eta \rightarrow \infty$ when $x \rightarrow \infty$ or $t \rightarrow 0$. When $\alpha=0$, the limit $t \rightarrow 0$ requires $s \rightarrow 0$ and since $0 \leq x \leq s$ the co-ordinate $\eta=x-s \rightarrow 0$. So in this case the initial condition must coincide with the left hand boundary condition. Since initially the temperature is everywhere at $T(x, t)=G(\eta)=-1$, the condition $G(0)=-s_{t}$ then requires $s_{t}=1$.

The Stefan condition integrates immediately to give $s(t)=\nu t$ and this may be used to remove the time dependence in the boundary condition $G(0)=\nu$. Integrating the governing equation (38) and applying the boundary conditions in (39) leads to

$$
G=-1+(1-\nu) e^{-\nu \eta} .
$$

To determine the value of $\nu$ we now use the above solution and the definition $\nu=-G^{\prime}(0) / \beta$ to show that $\nu=1-\beta$ and hence

$$
G=-1+\beta e^{-\eta(1-\beta)}, \quad s=(1-\beta) t,
$$

which is obviously a travelling wave. Since the front propagates in the positive $\eta$ direction, $s_{t}>0$, the solution is restricted to values $\beta<1$.

The travelling wave solution describes a constant shape that propagates with time: the temperature and temperature gradient are constant at the moving front $G(0)=-1+\beta$, $G_{\eta}(0)=-\beta(1-\beta)$. However, we noted above that for $T_{I}(t) \neq 0$ the initial condition is $s_{t}(0)=1$ but here we have $s_{t}(0)=1-\beta \neq 1$ : in other words, we do not satisfy the initial condition. The travelling wave solution is commonly quoted as an exact solution to Stefan problems but in this case it may only be considered as a large time solution and this will become clear when we compare against the numerics. With this in mind we should not apply $s(0)=0$ and the results in the original variables are

$$
T=-1+\beta e^{-(1-\beta)(x-s)}, \quad s=(1-\beta) t+C_{0}, \quad s_{t}=(1-\beta) .
$$

Case $\beta>1$ : $\quad$ For $\beta>1$ we can make progress by applying a large time substitution $t=\tau / \epsilon$ to (21)-(22) but now with $\alpha=1 / 2$. The Stefan condition (22b) then implies scaling $s=r / \epsilon^{1 / 2}$ and the governing equations are

$$
\begin{aligned}
G^{\prime \prime} & =-\left(\nu+\frac{\eta}{2}\right) G^{\prime}, \quad 0<\eta<\infty \\
G(0) & =-\epsilon^{1 / 2} r_{\tau}, \quad \tau^{1 / 2} r_{\tau}=\nu,\left.\quad G\right|_{\eta \rightarrow \infty}=-1 .
\end{aligned}
$$

Note, in the previous example we simply solved for $G$ and then found we could not apply the initial condition $s(0)=0$, hence suggesting a large time solution. Here we begin under the assumption of large time and so do not impose $s(0)=0$. The Stefan condition then immediately leads to $r=2 \nu \sqrt{\tau}+c_{0}$, where $c_{0}$ is an integration constant. The small parameter in the boundary condition suggests a series solution $G=G_{0}+$ $\mathcal{O}\left(\epsilon^{1 / 2}\right)$. The leading order problem is then simply the $\alpha=1 / 2$ case without kinetic undercooling (25)-(26) but the initial condition no longer applies. Viewed from another angle we can interpret the no kinetic undercooling problem as a large time leading order approximation to the kinetic undercooling problem. The first order problem has a timedependent boundary condition and so, as for the small time solution in Section $\S 3.2$, only the leading order problem is consistent with the current similarity transformation.

Writing the solution back in the original variables gives

$$
T \approx-1+\frac{\operatorname{erfc}\left(x /(2 \sqrt{t})-C_{1}\right)}{\operatorname{erfc}(\nu)}, \quad s \approx 2 \nu \sqrt{t}+C_{1}, \quad s_{t} \approx \frac{\nu}{\sqrt{t}} .
$$


Case $\beta=1$ : It is clear that this intermediate case cannot be found from the arguments given above and the analysis turns out to be more delicate. We therefore study it in more detail.

Instead of the similarity transformation given in (18) we define

$$
\eta=\frac{y}{t^{\alpha}}, \quad F(y, t)=G(\eta, t) .
$$

The governing equations (14)-(16) are now

$$
\begin{aligned}
\frac{\partial^{2} G}{\partial \eta^{2}} & =t^{2 \alpha} \frac{\partial G}{\partial t}-\left(\alpha \eta t^{2 \alpha-1}+t^{\alpha} s_{t}\right) \frac{\partial G}{\partial \eta}, \quad 0<\eta<\infty \\
\left.G\right|_{\eta=0} & =-s_{t},\left.\quad G\right|_{\eta \rightarrow \infty} \rightarrow-1, \quad t^{\alpha} s_{t}=-\left.\frac{\partial G}{\partial \eta}\right|_{\eta=0},
\end{aligned}
$$

again we do not impose $s(0)=0$.

We apply a large time substitution

$$
t=\frac{\tau}{\epsilon}, \quad s=\frac{r}{\epsilon^{\gamma}}, \quad G(\eta, t)=H(\eta, \tau),
$$

to equations (47)-(48), for the moment leaving the exponents $\alpha, \gamma$ unknown. Then the Stefan condition in (48) becomes

$$
\tau^{\alpha} r_{\tau}=\left.\epsilon^{\alpha+\gamma-1} \frac{\partial H}{\partial \eta}\right|_{\eta=0}
$$

and so we must have $\gamma=1-\alpha$ for the front to move (at leading order). Hence, the governing equations are

$$
\begin{aligned}
\frac{\partial^{2} H}{\partial \eta^{2}} & =\tau^{2 \alpha} \epsilon^{1-2 \alpha} \frac{\partial H}{\partial \tau}-\left(\alpha \eta \tau^{2 \alpha-1} \epsilon^{1-2 \alpha}+\tau^{\alpha} r_{\tau}\right) \frac{\partial H}{\partial \eta}, \quad 0<\eta<\infty \\
\left.H\right|_{\eta=0} & =-\epsilon^{\alpha} r_{\tau},\left.\quad H\right|_{\eta \rightarrow \infty} \rightarrow-1, \quad \tau^{\alpha} r_{\tau}=-\left.\frac{\partial H}{\partial \eta}\right|_{\eta=0} .
\end{aligned}
$$

From examining (50) and (51a) it is clear that we require $0<\alpha<1 / 2$, in order that $\epsilon^{1-2 \alpha}, \epsilon^{\alpha} \rightarrow 0$ as $\epsilon \rightarrow 0$. We now introduce expansions

$$
H(\eta, \tau)=H_{0}(\eta)+\epsilon^{1-2 \alpha} \tau^{\theta} H_{1}(\eta)+\ldots, \quad r(\tau)=r_{0}(\tau)+\epsilon^{1-2 \alpha} r_{1}(\tau)+\ldots,
$$

where again $\theta$ is left unspecified. Substituting these expansions into (50)-(51) gives the leading order problem

$$
\begin{aligned}
H_{0}^{\prime \prime}(\eta) & =-\nu_{0} H_{0}^{\prime}(\eta), \quad 0<\eta<\infty \\
H_{0}(0) & =0,\left.\quad H_{0}\right|_{\eta \rightarrow \infty} \rightarrow-1, \quad \tau^{\alpha} r_{0 \tau}=\nu_{0},
\end{aligned}
$$

where $\nu_{0}=-H_{0}^{\prime}(0)$. This has solution

$$
H_{0}(\eta)=-1+e^{-\nu_{0} \eta}, \quad r_{0}=\frac{\nu_{0}}{1-\alpha} \tau^{1-\alpha}+c_{0}, \quad \beta=1 .
$$

Note, the definition of $\nu_{0}=-H_{0}^{\prime}(0)$ is automatically satisfied and provides no information. We must then look to the higher order terms to determine $\nu_{0}$. Neglecting the leading order terms in the boundary condition for $\left.H\right|_{\eta=0}$ leads to the balance $\epsilon^{1-2 \alpha} \tau^{\theta} H_{1}(0)=-\epsilon^{\alpha} r_{0 \tau}$. To make the terms balance we choose $\alpha=1 / 3$. Thus the $\mathcal{O}\left(\epsilon^{1 / 3}\right)$ problem is

$$
\begin{aligned}
\tau^{\theta} H_{1}^{\prime \prime}(\eta) & =-\frac{\eta}{3} \tau^{-1 / 3} H_{0}^{\prime}(\eta)-\nu_{0} \tau^{\theta} H_{1}^{\prime}(\eta)-\tau^{1 / 3} r_{1 \tau} H_{0}^{\prime}(\eta), \quad 0<\eta<\infty \\
\tau^{\theta} H_{1}(0) & =-r_{0 \tau},\left.\quad H_{1}\right|_{\eta \rightarrow \infty} \rightarrow 0, \quad \tau^{1 / 3} r_{1 \tau}=-\tau^{\theta} H_{1}^{\prime}(0) .
\end{aligned}
$$


To remove $\tau$ from the equations requires setting $\theta=-1 / 3$. Thus (56)-(57) reduce to

$$
\begin{aligned}
& H_{1}^{\prime \prime}(\eta)=-\frac{\eta}{3} H_{0}^{\prime}(\eta)-\nu_{0} H_{1}^{\prime}(\eta)-\nu_{1} H_{0}^{\prime}(\eta), \quad 0<\eta<\infty \\
& H_{1}(0)=-\nu_{0}, \quad \tau^{2 / 3} r_{1 \tau}=\nu_{1},\left.\quad H_{1}\right|_{\eta \rightarrow \infty} \rightarrow 0,
\end{aligned}
$$

where $\nu_{1}=-H_{1}^{\prime}(0)$. The solution to this system is

$$
H_{1}=-\frac{1}{6 \nu_{0}^{2}}\left[2+6 \nu_{0}^{2} \nu_{1} \eta+2 \nu_{0} \eta+\nu_{0}^{2} \eta^{2}\right] e^{-\nu_{0} \eta}
$$

Substituting for $H_{1}(0)$ into (59a) gives an equation to determine $\nu_{0}$

$$
\nu_{0}=\left(\frac{1}{3}\right)^{1 / 3}
$$

Finally we may write down the position of the moving front

$$
r_{0}=\left(\frac{9}{8}\right)^{1 / 3} \tau^{2 / 3}+c_{0}
$$

Note, the dependence $r \sim \tau^{2 / 3}$ is quoted in $[6,7,10,11]$ (without the multiplicative constant). Finally, in the original notation we have

$$
T \approx-1+\exp \left(\frac{x-s}{(3 t)^{1 / 3}}\right), \quad s \approx\left(\frac{9}{8}\right)^{1 / 3} t^{2 / 3}+C_{2}, \quad s_{t} \approx(3 t)^{-1 / 3} .
$$

\subsection{Nonlinear kinetic undercooling}

Theoretically, the Neumann solution holds for any $\beta>1$. Adopting the linear approximation to the $s_{t}\left(T_{I}\right)$ relation permits the solution domain to be extended to include $\beta \leq 1$. Taking the values for $L_{m}, c_{l}$ for salol given in Table 1 indicates $\beta \rightarrow 1$ as $\Delta T \rightarrow 57 \mathrm{~K}$ and this is where the Neumann solution predicts the velocity tends to infinity. However, if we look again at Figure 1b) it is clear that the velocity is in fact significantly below its maximum value of $4.5 \times 10^{-5} \mathrm{~m} / \mathrm{s}$. Further, the linear model is only valid for $\Delta T<10 \mathrm{~K}$, that is for $\beta>5.7$. For copper a similar argument indicates the linear model holds for $\beta>3.95$. Consequently, for relatively large values of $\beta$ neither the Neumann or linear approximations will provide physically realistic solutions and we must deal with the full nonlinear relation (12).

Key to the similarity solutions of the previous section was the ability to remove the time dependence from the conditions at $x=s(t)$. The nonlinear relation (12) makes this a much more difficult task and so in this section we limit our analysis to small time and travelling wave solutions. In the subsequent section we will then introduce an accurate form of heat balance integral method which permits approximate solutions for further cases.

\subsubsection{Small time solutions}

Using the previous definitions of $\eta, G$ as given in Section $\S 3.2$ we obtain equations (29)(30) with the only difference being that in the boundary condition (30a) we replace the right hand side with $T_{I}$. This relation is then used in equation (12) to give

$$
s_{t}=\left[1-t^{1 / 2} G(0)\right] \exp \left\{\frac{Q\left[-1+t^{1 / 2} G(0)\right]}{P+\left[-1+t^{1 / 2} G(0)\right]}\right\} .
$$

We now make the small time substitution $t=\epsilon \tau, s=\epsilon r$, and expand the exponential term using a Taylor series expansion. This brings us to the leading order problem specified by equations $(33,34)$ but with the condition $r_{0 \tau}=1$ replaced by

$$
r_{0 \tau}=\exp \left(-\frac{Q}{P-1}\right) \text {. }
$$


This has solution

$$
G_{0}=\beta \exp \left(-\frac{Q}{P-1}\right)\left[\frac{2}{\sqrt{\pi}} e^{-\eta^{2} / 4}-\eta \operatorname{erfc}\left(\frac{\eta}{2}\right)\right], \quad r_{0}=\tau \exp \left(-\frac{Q}{P-1}\right) .
$$

As before we cannot find $G_{1}$ but can determine an expression for $r_{1}$, namely

$$
r_{1}=-\exp \left(-\frac{2 Q}{P-1}\right)\left[1-\frac{Q P}{(P-1)^{2}}\right] \tau^{3 / 2} \frac{4 \beta}{3 \sqrt{\pi}} .
$$

Writing the solution back in the original variables leads to

$$
\begin{aligned}
T & \approx-1+t^{1 / 2} \beta \exp \left(-\frac{Q}{P-1}\right)\left[\frac{2}{\sqrt{\pi}} e^{-\frac{(x-s)^{2}}{4 t}}-\left(\frac{x-s}{t^{1 / 2}}\right) \operatorname{erfc}\left(\frac{x-s}{2 t^{1 / 2}}\right)\right], \\
s & \approx \exp \left(-\frac{Q}{P-1}\right) t\left\{1-\exp \left(-\frac{Q}{P-1}\right)\left[1-\frac{Q P}{(P-1)^{2}}\right] \frac{4 \beta}{3 \sqrt{\pi}} t^{1 / 2}\right\}, \\
s_{t} & \approx \exp \left(-\frac{Q}{P-1}\right)\left\{1-\exp \left(-\frac{Q}{P-1}\right)\left[1-\frac{Q P}{(P-1)^{2}}\right] \frac{2 \beta}{\sqrt{\pi}} t^{1 / 2}\right\} .
\end{aligned}
$$

\subsubsection{Large time solutions}

Case $\beta<1$ : The travelling wave analysis in Section $\S 3.2$ easily translates to the current problem. We find

$$
G=-1+\left(1+T_{I}\right) e^{-\nu \eta},
$$

where $T_{I}=\beta-1$. The wave speed $\nu$, which varies with $T_{I}$, follows from substituting $s_{t}=\nu$ into equation $(12)$

$$
\nu=s_{t}=(1-\beta) \exp \left(\frac{Q(\beta-1)}{P+\beta-1}\right) .
$$

The temperature in the original variables is

$$
T=-1+\beta \exp (-\nu(x-s))
$$

and the position and velocity of the freezing front are

$$
s=(1-\beta) \exp \left(\frac{Q(\beta-1)}{P+\beta-1}\right) t+C_{0}, \quad s_{t}=(1-\beta) \exp \left(\frac{Q(\beta-1)}{P+\beta-1}\right) .
$$

As in the linear case, the above travelling wave solution is restricted to values $\beta<1$ and, since it cannot satisfy the initial condition, should be considered a large time approximation.

\section{Solution with the HBIM}

The heat balance integral method (HBIM) introduced by Goodman [18] is a well-known approximate method for solving Stefan problems. The basic idea behind the method is to approximate the temperature profile, usually with a polynomial, over some distance $\delta(t)$ known as the heat penetration depth. The heat equation is then integrated to determine a simple ordinary differential equation for $\delta$. The solution of this equation, coupled with the Stefan condition then determines the temperature and position $s(t)$. The popularity of the HBIM is mainly due to its simplicity. However, in its original form there are a number of drawbacks, primarily a lack of accuracy for certain problems but also the rather arbitrary choice of approximating function, see [19] for a more detailed description of the method and problems. Recently a number of variants of the HBIM have been developed which address the issues and have led to simple solution methods that, over physically realistic parameter ranges, have proved more accurate than second order perturbation solutions 
$[20,21,22,23]$. For the current study we will use the HBIM to permit us to make further analytical progress in the case of large supercooling.

For the one-phase semi-infinite problem with large supercooling the HBIM proceeds as follows. For $t>0$ the temperature decreases from $T(s, t)=T_{I}$ to -1 as $x \rightarrow \infty$. With the HBIM the temperature profile is specified over a finite distance $x \in[s, \delta]$, where the 'heat penetration depth' $\delta$ is defined as the position beyond which the temperature rise is negligible. This leads to the boundary conditions $T(\delta, t)=-1, T_{x}(\delta, t)=0$ and $\delta(0)=0$. The simplest polynomial profile satisfying these conditions, along with $T(s, t)=T_{I}(t)$, is given by the function

$$
T=-1+\left(1+T_{I}\right)\left(\frac{\delta-x}{\delta-s}\right)^{n} .
$$

In the original HBIM the value $n=2$ was employed, although other values have been used in later studies (often motivated by numerical solutions), see [19]. For now we leave it unspecified. The heat balance integral is determined by integrating the heat equation (8) over the spatial domain, leading to

$$
\frac{\mathrm{d}}{\mathrm{d} t} \int_{s}^{\delta} T \mathrm{~d} x-\left.T\right|_{x=\delta} \delta_{t}+\left.T\right|_{x=s} s_{t}=\left.T_{x}\right|_{x=\delta}-\left.T_{x}\right|_{x=s} .
$$

Substituting the expression for $T$ from (75) gives

$$
\frac{\mathrm{d}}{\mathrm{d} t}\left[\frac{\left(T_{I}-n\right)(\delta-s)}{n+1}\right]+\delta_{t}+T_{I} s_{t}=\frac{n\left(1+T_{I}\right)}{\delta-s} .
$$

This involves the unknowns, $\delta(t), s(t)$. A further equation comes from the Stefan condition

$$
\beta s_{t}=\frac{n\left(1+T_{I}\right)}{\delta-s}
$$

and the system is closed with equation (12). The exponent $n$ is also unknown and this is determined through Myers' method, described in [22, 23]. If we define $f(x, t)=T_{t}-T_{x x}$, then the HBIM may be specified through the integral $\int_{s}^{\delta} f d x=0$. The modification suggested in $[22,23]$ was to choose $n$ to minimise the least-squares error $E_{n}=\int_{s}^{\delta} f^{2} d x$. This leads to significant improvements in the accuracy of the HBIM as well as providing an error measure that does not require knowledge of an exact or numerical solution. For certain problems, for example when the boundary conditions are time-dependent, $n$ may vary with time. To keep the method simple $n$ is then set to its initial value, since this is where the greatest error $E_{n}$ usually occurs. A subsequent refinement to this method, called the Combined Integral Method (CIM), was developed by Mitchell \& Myers [21, 24] which provides a more consistent way to deal with cases where $n$ is time-dependent.

The standard HBIM is often criticized due to a lack of accuracy. To indicate the accuracy of these new methods we point out that in [24] the CIM, second order large $\beta$ and leading order small $\beta$ perturbation solutions are compared against the exact solution for a two-phase supercooled Stefan problem. For $\beta \in[0.012,51.5]$ the CIM is the most accurate method with a percentage error in the front velocity in the range $[0.1,0.4] \%$. In the range $\beta \in[1,51.5]$ the error for the 2 nd order perturbation varies almost linearly between 100 and $0.4 \%$ (for $\beta<1$ the error is off the graph). Over the whole range plotted in [24, Fig. 5], $\beta \in\left[10^{-4}, 10^{2}\right]$, the CIM error is a decreasing function of $\beta$ with a maximum when $\beta=10^{-4}$ of around $0.44 \%$. Given that the polynomial exponents only depend on $\beta$ we expect Myers' method to be even more accurate than the CIM and so in the following, for simplicity, we will restrict $n$ to be independent of time and so use Myers' method.

\subsection{Linear kinetic undercooling}

For the case of linear kinetic undercooling, $T_{I}(t)=-s_{t}$, and so we can use the Stefan condition (78) to eliminate $\delta$ from (77) and so derive an equation depending solely on 
$s(t)$. Assuming $n$ is constant we obtain the second order ODE

$$
s_{t t}=\frac{(n+1) \beta s_{t}^{3}\left(1-\beta-s_{t}\right)}{n\left(1-s_{t}^{2}\right)} .
$$

Hence the HBIM has reduced the initial Stefan problem to one of solving a single ordinary differential equation for $s$. This is obviously a much simpler prospect than solving the full Stefan problem. Once $s$ is known the interface temperature is determined by $T_{I}=-s_{t}$, $\delta$ is given by equation (77) and the temperature profile follows from (75). As discussed earlier the initial conditions are $s(0)=0, s_{t}(0)=1$. Equation (79) is easily solved using the Matlab routine ode15s. In this case, the error minimisation process leads to $n \approx 3.57$. The condition $s_{t}(0)=1$ leads to an initial singularity in acceleration. Motivated by the previous small time solution we assume a form $s_{t}=1+B t^{\alpha}$ when $t \ll 1$ which leads to $s_{t}=1-\sqrt{\beta^{2}(n+1) / n} t^{1 / 2}$ and hence $s=t-(2 / 3) \sqrt{\beta^{2}(n+1) / n} t^{3 / 2}$. These forms are then used as the initial conditions for the numerical calculation starting at some time $t=t_{0} \ll 1$.

Note, this is not the first time that an HBIM has been applied to kinetic undercooling problems. Charach \& Zaltzman [7] studied the linear case employing the error function profile

$$
T=-1+\left(1+T_{I}\right) \operatorname{erfc}[c(x-s)],
$$

where $c$ is an unknown time-dependent coefficient to be determined. This form was motivated by the $T_{I}=0$ case of equation (27). The solutions obtained in this manner exhibit the same large time behaviours discussed earlier although by comparing to the numerical results we found this profile to be significantly less accurate than that using Myers' method. It also requires $c$ to be determined and $c(0)$ turns out to be infinite. For these reasons we do not show this solution on any plots.

\subsection{Nonlinear kinetic undercooling}

For the case of large supercooling the appropriate expression for $T_{I}(t)$ is obtained from equation (12). Eliminating $\delta$ from (77) by means of the Stefan condition (78) we obtain

$$
\frac{2\left(1+T_{I}\right)}{s_{t}} \frac{d T_{I}}{d t}-\frac{\left(1+T_{I}\right)^{2}}{s_{t}^{2}} s_{t t}=\frac{(n+1) \beta\left(\beta-1-T_{I}\right)}{n} s_{t} .
$$

The above equation suggests making the change of variable $y=s_{t}$, which leads to

$$
\frac{\mathrm{d} T_{I}}{d t}=\frac{\left(1+T_{I}\right)}{2 y} \frac{\mathrm{d} y}{d t}+\frac{(n+1) \beta\left(\beta-1-T_{I}\right) y^{2}}{2 n\left(1+T_{I}\right)} .
$$

A second equation can be obtained by taking the time derivative of (12)

$$
\frac{\mathrm{d} y}{d t}=-\exp \left(\frac{Q T_{I}}{P+T_{I}}\right)\left[1+\frac{Q P T_{I}}{\left(P+T_{I}\right)^{2}}\right] \frac{\mathrm{d} T_{I}}{d t} .
$$

Equations (82) and (83), together with the definition $y=s_{t}$, constitute a system of three nonlinear first order ODEs that can be easily solved with the Matlab routine ode15s. The initial conditions for this system are $T_{I}(x, 0)=-1, y(0)=\exp (-Q /(P-1))$ and $s(0)=0$. Again, the exponent $n$ is determined by minimizing $E_{n}$ for $t \approx 0$ giving $n \approx 3.61$.

\subsection{Asympotic analysis within the HBIM formulation}

\subsubsection{Linear kinetic undercooling}

Applying a large time subsitution allows us to examine the solution behaviour analytically and in particular make comparison with earlier solution forms. Firstly, we write $t=\tau / \epsilon$ and $s=r / \epsilon^{\gamma}$ and equation (79) becomes

$$
\epsilon^{2-\gamma} r_{\tau \tau}=\frac{(n+1) \beta \epsilon^{3(1-\gamma)} r_{\tau}^{3}\left(1-\beta-\epsilon^{1-\gamma} r_{\tau}\right)}{n\left(1-\epsilon^{2(1-\gamma)} r_{\tau}^{2}\right)}
$$


The obvious balance comes from setting $2-\gamma=3(1-\gamma)$, which gives $\gamma=1 / 2$, and so (84) reduces to

$$
r_{\tau \tau} \approx \frac{(n+1) \beta(1-\beta) r_{\tau}^{3}}{n} \quad \Rightarrow \quad r_{\tau}^{-2} \approx \frac{2(n+1) \beta(\beta-1) \tau}{n}+c_{0} .
$$

To ensure $r_{\tau}>0$ requires $\beta>1$. Since all solutions with $\beta \geq 1$ have $s_{t} \rightarrow 0$ (or equivalently $\left.T_{I} \rightarrow 0\right)$ as $t \rightarrow 0$ the constant $c_{0}=0$. This indicates $s_{t} \approx \sqrt{n /(2(n+1) \beta(\beta-1) t)}$ and is consistent with the large time solution given in Section $\S 3.2$ for $\beta>1$.

A second balance comes from setting $\gamma=1$ in (84). Then the right hand side is dominant and so $r_{\tau}=1-\beta$, giving $s_{t}=1-\beta$ (and so requiring $\beta<1$ ). This is consistent with the travelling wave solution, valid for $\beta<1$, found in Section $\S 3.2$.

A third reduction is obtained by first setting $\beta=1$ in equation (84)

$$
\epsilon^{2-\gamma} r_{\tau \tau}=-\frac{(n+1) \epsilon^{4(1-\gamma)} r_{\tau}^{4}}{n\left(1-\epsilon^{2(1-\gamma)} r_{\tau}^{2}\right)}
$$

Then balancing both sides gives $2-\gamma=4(1-\gamma)$, or $\gamma=2 / 3$, leading to

$$
r_{\tau \tau} \approx-\frac{(n+1) r_{\tau}^{4}}{n} \quad \Rightarrow \quad r_{\tau} \approx\left(\frac{3(n+1) \tau}{n}\right)^{-1 / 3} .
$$

Thus

$$
s_{t} \approx\left(\frac{3(n+1) t}{n}\right)^{-1 / 3} \quad \Rightarrow \quad s \approx\left(\frac{9 n}{8(n+1)}\right)^{1 / 3} t^{2 / 3}
$$

Again the constants of integration have been set to zero to achieve the appropriate behaviour as $t \rightarrow \infty$. This is the behaviour predicted by the large time, $\beta=1$ analysis of Section $\S 3.2$.

From the above we see that the HBIM formulation allows us to easily capture the three forms of solution behaviour determined in $\S 3.2 .2$.

\subsubsection{Nonlinear kinetic undercooling}

As in the previous section we may make analytical progress in the large time limit by setting $t=\tau / \epsilon, s=r / \epsilon^{\gamma}$ and also $T_{I}=\epsilon^{\theta} U$, where $\gamma, \theta \geq 0$. Equation (12) then becomes

$$
\epsilon^{1-\gamma} r_{\tau}=-\epsilon^{\theta} U \exp \left(\frac{Q \epsilon^{\theta} U}{P+\epsilon^{\theta} U}\right)
$$

This equation clearly shows that for the front to move $\theta=1-\gamma$. Equation (81) becomes

$$
\epsilon \frac{2\left(1+\epsilon^{1-\gamma} U\right)}{r_{\tau}} \frac{d U}{d \tau}-\epsilon^{\gamma} \frac{\left(1+\epsilon^{1-\gamma} U\right)^{2}}{r_{\tau}^{2}} r_{\tau \tau}=\epsilon^{1-\gamma} \frac{(n+1) \beta\left(\beta-1-\epsilon^{1-\gamma} U\right)}{n} r_{\tau} .
$$

We note that the first term in (90) will never be dominant for any value of $\gamma \in[0,1]$. So, in fact,

$$
-\epsilon^{\gamma} \frac{\left(1+\epsilon^{1-\gamma} U\right)^{2}}{r_{\tau}^{2}} r_{\tau \tau} \approx \epsilon^{1-\gamma} \frac{(n+1) \beta\left(\beta-1-\epsilon^{1-\gamma} U\right)}{n} r_{\tau}, \quad \forall \gamma
$$

Moreover, we realize that the exponential in (89) affects the leading order term only when $\gamma=1$, otherwise $r_{\tau}=-U+\mathcal{O}\left(\epsilon^{1-\gamma}\right)$, i.e. the linear case is retrieved, and so we find the same balances as in the previous section. First, for $\gamma=1 / 2$ (91) reduces to (85) valid for $\beta>1\left(s_{t} \sim t^{-1 / 2}\right)$. Second, setting $\beta=1$, we find that $\gamma=2 / 3$ and (91) reduces to (88) $\left(s_{t} \sim t^{-1 / 3}\right)$. Finally, for $\gamma=1$ the right hand side of (91) is dominant and we find $U=T_{I}=\beta-1$. Then $s_{t}$ is described by the travelling wave solution (72) which requires $\beta<1$. There is also the possibility of setting $\gamma=0$ but this implies $T_{I}=\epsilon U \ll 1$ which is not consistent with the specification of large supercooling. 


\subsubsection{Discussion}

The equations obtained through the HBIM formulation for the linear case are easily analysed to determine the three time dependencies found at large times by previous authors. In the nonlinear case the asymptotic analysis may no longer be applied, however the HBIM formulation indicates the three same solution forms. When $\beta \geq 1$, as $t \rightarrow \infty$ the velocity $s_{t} \rightarrow 0$ and so $T_{I} \rightarrow 0$. Consequently we should expect the linear and nonlinear cases to coincide. The numerics of the following section confirms this. When $\beta<1$ the value of $s_{t}$ is constant and differs for the linear and nonlinear cases.

\section{Results}

We now present a set of results for the various scenarios discussed in $\S 3$ and $\S 4$. The asymptotic and HBIM results are compared with a numerical scheme similar to that developed by Mitchell \& Vynnycky [25, 26]. This uses the Keller box scheme, which is a second order accurate finite-difference method, and has been successfully applied to several moving boundary problems. The boundary immobilisation transformation at the end of $\S 2$, along with a small time analysis, ensures that the correct starting solution is used in the numerical scheme. In all examples we use parameter values for copper, hence $Q \approx 3.5811$. The value of $\beta$ and $P$ depend on $\Delta T$ hence the plots with $\beta=0.7,1,1.5$ correspond to $P \approx 2.417,3.453,5.179$ respectively.

For clarity we present the results in three subsections: in the first we compare numerics, HBIM and asymptotic solutions for the case of linear kinetic undercooling, then repeat this for nonlinear undercooling, and finally we compare results obtained through the two undercooling models and the classical Neumann solution.

\section{$5.1 \quad$ Linear kinetic undercooling}
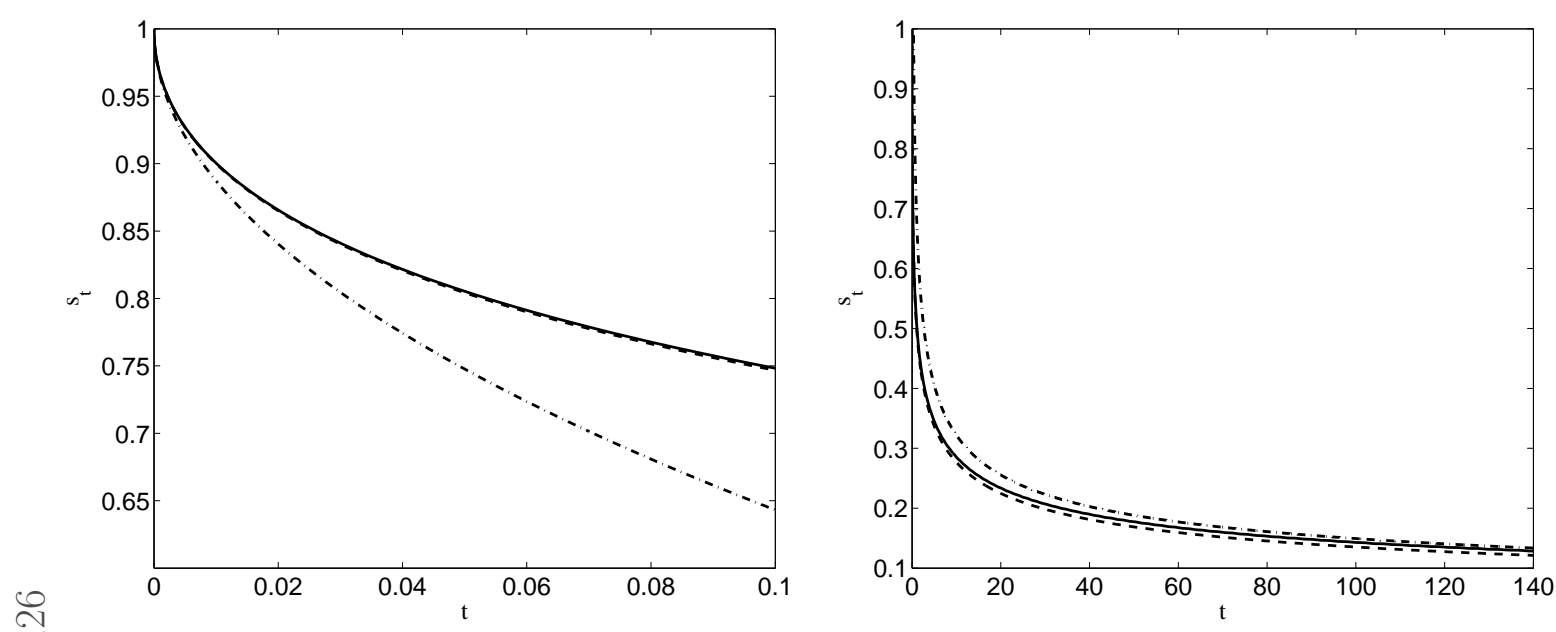

Figure 2: Linear kinetic undercooling with a) $t \in[0,0.1]$, b) $t \in[0,140]$. The sets of curves denote the numerical solution (solid line), HBIM (dashed) and small and large time asymptotics (dash-dotted) results for the interface velocity when $\beta=1$.

In Figure 2 we compare the results of the numerical solution (solid line), the HBIM (dashed) defined by equation (79) and the small and large time asymptotics (dash-dot) for the case $\beta=1$. The value of the exponent used in the HBIM was $n=3.57$ which was determined by minimising the least-squares error. The left-hand figure shows the solutions for $t \in[0,0.1]$. In this range the numerical and HBIM solutions are virtually indistinguishable whilst the small time asymptotic solution is only accurate for the very 
initial stage. The asymptotic solution does have a time restriction since the leading order term must be significantly greater than the first order. In fact a more rigorous bound is imposed by the restriction that $s_{t}>0$ thus requiring $t<\pi /\left(4 \beta^{2}\right)$ and so the smaller the value of $\beta$ the longer the solution is valid. The right hand figure shows a comparison of the numerics, HBIM solution and large time asymptotics carried on until $t=140$. Again the HBIM is close to the numerics for all of the range. The large time asymptotic solution improves as $t$ increases and for $t$ approximately greater than 80 becomes more accurate than the HBIM.
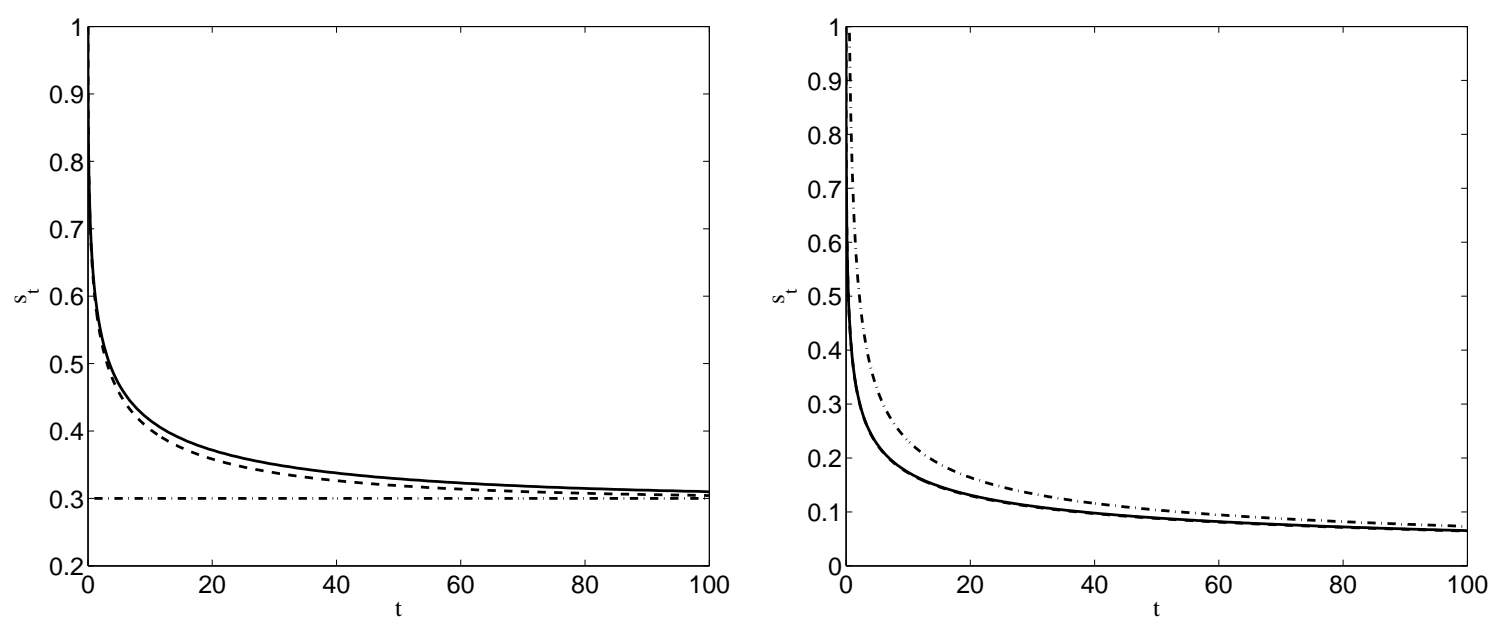

Figure 3: Linear kinetic undercooling with $t \in[0,100]$ and a) $\beta=0.7, b) \beta=1.5$. The sets of curves denote the numerical solution (solid line), HBIM (dashed) and large time asymptotics (dash-dotted) results for the interface velocity.

Figure 3 displays two sets of results demonstrating the two other forms of behaviour, with $\beta<1$ and $\beta>1$. The left hand figure is for $\beta=0.7$. For large times the solution is a travelling wave. The large time asymptotic result is therefore a straight line corresponding to the wave speed $s_{t}=1-\beta=0.3$. As mentioned in $\S 3.2 .2$, the travelling wave does not match the initial conditions, which the HBIM and numerics correctly capture, and so must be classified as a large time solution. Even at $t=100$ the travelling wave speed is around $3 \%$ below the numerical solution.

For $\beta>1$ the large time asymptotic solution (at leading order) reduces to the no kinetic undercooling (or Neumann) solution. The velocity is represented by (45c) and it is shown in the right hand plot in Figure 3 for $\beta=1.5$ together with numerical and HBIM results (which again are virtually indistinguishable). At $t=100$ the difference between numerics and asymptotics is around 10\%. However, if we increase $\beta$ to 2 the error at $t=100$ reduces to $4 \%$.

\subsection{Nonlinear kinetic undercooling}

In Figures 4, 5 we demonstrate different solution behaviours for the nonlinear kinetic undercooling case. In contrast to the linear examples we do not have large time asymptotic solutions for $\beta \leq 1$ and only show HBIM and numerical solutions for those cases. The HBIM solution is obtained by integrating (82)-(83) using Matlab routine ode15s.

The left hand plot in Figure 4 shows the small time behaviour with $\beta=1$, when $t \in[0,0.1]$. Again the HBIM (with $n=3.57$ ) appears to be very accurate, whilst the asymptotic solution slowly loses accuracy. As before this is bounded by a time restriction, $t \ll \pi / 4 \beta^{2} \exp [2 Q /(1-P)]\left[1-Q P /(P-1)^{2}\right]^{2}$. Decreasing $\beta$ would improve the accuracy of the asymptotic solution. The HBIM solution has the initial value $s_{t}=\exp (-Q /(P-1))$, which in this case gives $s_{t}(0) \approx 0.232$. An important difference between this solution 

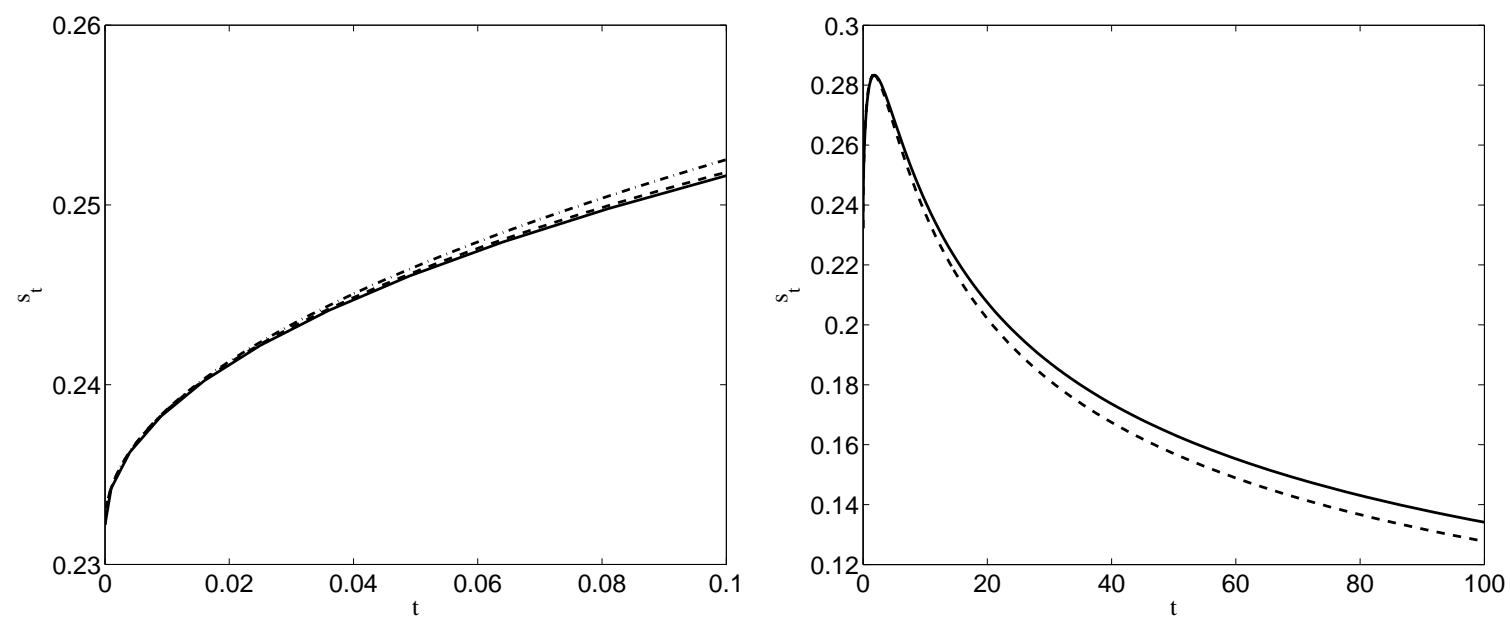

Figure 4: Nonlinear kinetic undercooling with a) $t \in[0,0.1]$, b) $t \in[0,100]$. The sets of curves denote the numerical solution (solid line), HBIM (dashed) and small time asymptotics (dash-dotted) results for the interface velocity when $\beta=1$.

and the zero and linear undercooling cases is that the speed now increases with time. Referring to Figure 1, this indicates that this value of Stefan number requires $T_{m}-T_{I}$ such that we begin to the right of the peak in the $s_{t}(\Delta T)$ curve. As time proceeds and $T_{I}$ approaches $T_{m}$ we will move to the left and so observe an initial increase in $s_{t}$ followed by a decrease as we pass the peak. This behaviour is apparent in the right hand plot of Figure 4, which shows the numerical and HBIM solution for $t \in[0,100]$. For $t \in[0,5]$ (approximately) $s_{t}$ increases to a maximum of just above 0.28 and then slowly decreases with the $t^{-1 / 3}$ behaviour predicted in $\S 4.3$.
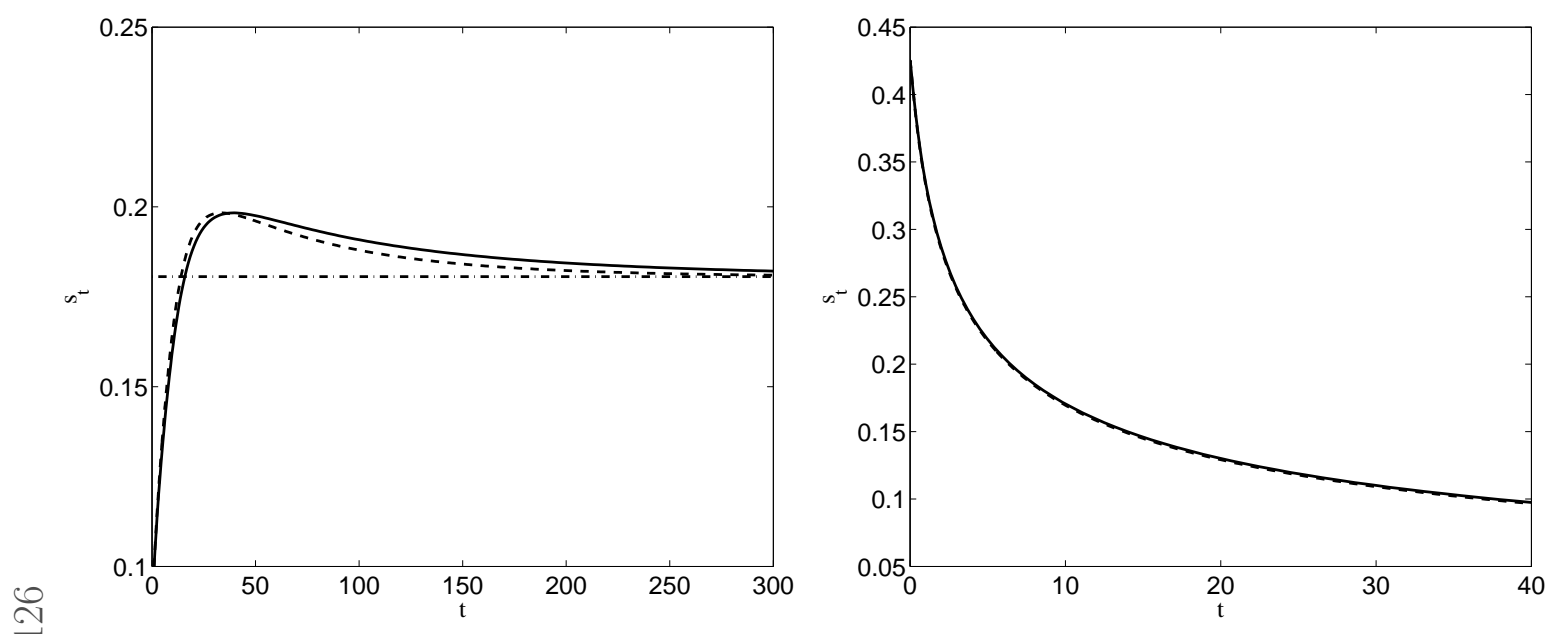

Figure 5: Nonlinear kinetic undercooling with a) $\beta=0.7, t \in[0,100]$ and b) $\beta=1.5$, $t \in[0,40]$. The sets of curves denote the numerical (solid line) HBIM (dashed) and, when $\beta=0.7$, asymptotic (dash-dot) solutions for the interface velocity.

Figure 5 displays results for $\beta=0.7,1.5$ for $t \in[0,300],[0,40]$ respectively. The left hand figure shows that the HBIM is always close to the numerical solution and that the travelling wave result is only achieved after a very large time. Even after $t=300$ the large time asymptotic solution is $4 \%$ below the numerical solution. This approach to the travelling wave is much slower than in the linear case. The HBIM and numerical results, as in Figure 4, show an initial growth in $s_{t}$ followed by a decrease for $t>30$, again this 
may be attributed to starting from the right of the peak in the $s_{t}(\Delta T)$ graph and then moving to the left, across the peak as $t$ increases. The right hand figure contains the HBIM and numerical solutions for $\beta=1.5$. Obviously the curves are very close to each other. The larger value of $\beta$ indicates a lower value of $\Delta T$ than in the left hand plot and this means the degree of undercooling is always such that we remain to the left of the peak on the $s_{t}(\Delta T)$ graph. Consequently $s_{t}$ is a decreasing function of time and, as shown in $\S 4.3, s_{t} \propto t^{-1 / 2}$.

At the end of $\S 4$ it was mentioned that the two solution forms, with $\beta \geq 1$, must approach the linear kinetic undercooling forms since $T_{I}=-s_{t} \rightarrow 0$. Comparing Figures 2 and 4 shows the large time solutions for $\beta=1$ do coincide, similarly with the results when $\beta=1.5$ shown in the right side of Figures 3 and 5 . However, when $\beta<1$ then $T_{I}=1-\beta$ does not approach $T_{m}$ and so linear and nonlinear results for $\beta=0.7$, shown in the left side of Figures 3 and 5 , have different limits, $s_{t}=0.3$ and $s_{t} \approx 0.18$ respectively.

\subsection{Comparison of linear and nonlinear undercooling}
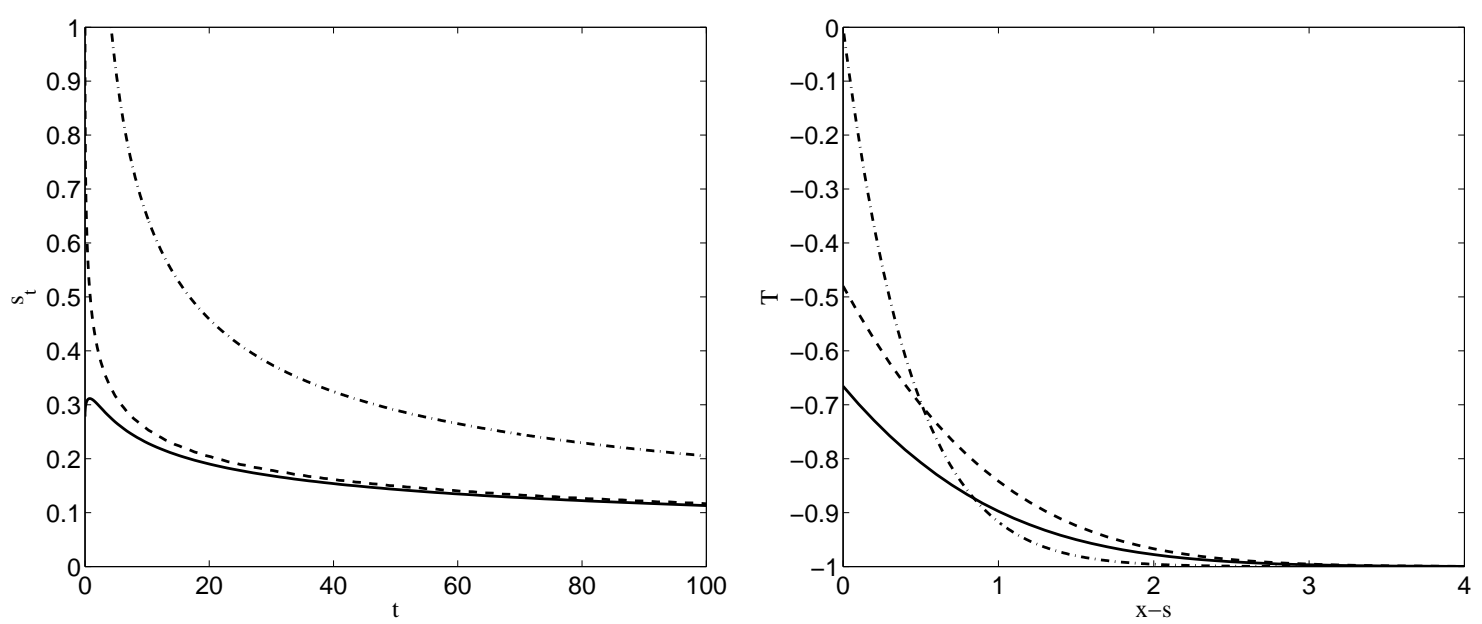

Figure 6: Comparison of velocities and temperatures $(a t t=1)$ predicted by the nonlinear (solid line), linear (dashed line) and Neumann (dot-dash) solutions for $\beta=1.1$.

In Figure 6 we show plots of the velocity $s_{t}$ for $t \in[0,100]$ and the temperature $T$ at $t=1$ for the case $\beta=1.1$. This $\beta$ value was chosen to permit the inclusion of the Neumann solution. On the plots the solid line represents the numerical solution of the nonlinear problem, the dashed line that of the linear case and the dot-dash line the Neumann solution.

The left hand plot shows the velocities $s_{t}$. The Neumann solution breaks down at $\beta=1$, however, it is clear from the curves that even for $\beta=1.1$ it is far from the solutions with undercooling. This indicates that, although the Neumann solution is accepted as valid down to $\beta=1$, it may be highly inaccurate and inappropriate for describing the solidification of a supercooled liquid. The choice $\beta=1.1$ means that we operate to the left of the peak in the $s_{t}(\Delta T)$ graph, where the linear approximation is close to the nonlinear curve. Consequently, the corresponding velocities shown on Figure 6 converge quite rapidly. However, for small times the linear case has much higher velocities which would result in a significantly higher prediction for $s$ than when using the nonlinear relation. Decreasing the value of $\beta$ causes the $s_{t}$ curves to diverge further.

The right hand plot shows the temperature profiles at $t=1$ as a function of the fixed boundary co-ordinate $y=x-s$. The Neumann solution (dash-dotted line) has a constant temperature $T=0$ at $x=s$ for all times, while the nonlinear (solid) and linear (dashed) solutions present a variable temperature at $x=s$ that tends to 0 as time increases. The 
differences observed between the temperature profiles become smaller for larger times and the solutions look almost the same at $t=100$.

\section{Conclusions}

In this paper we have investigated the one-phase one-dimensional Stefan problem with a nonlinear relation between the phase change temperature and solidification rate. In the limits of zero and linear (small) supercooling we reproduced the asymptotic behaviour found in previous studies. The asymptotics for the nonlinear regime proved more difficult and then our analysis was limited to small time solutions for arbitrary Stefan number and a travelling wave solution at large time (valid for $\beta<1$ ). A recent extension to the Heat Balance Integral Method was then applied to the system to reduce the problem to a single ordinary differential equation for the case of linear supercooling and two ordinary differential equations for the nonlinear case. Asymptotic techniques could then be applied to the ordinary differential equations to reproduce the various asymptotic behaviours found in the linear system. For the nonlinear system it turned out that the same behaviour could be found at large times and, given the proximity of the HBIM solution to numerical results we conclude that this reflects the behaviour of the full system.

Whilst asymptotic analysis is a popular method to analyse the solution form in various limits it may only be valid over a very small range. In contrast the HBIM solution was very close to the numerical solution and in general proved more accurate than the small and large time asymptotics. In the nonlinear case, where the asymptotic solutions were not available for all cases the HBIM equations could still be analysed to predict the solution behaviour. This indicates that the new accurate version of the HBIM is a useful tool in analysing this type of problem.

An interesting point concerns the Neumann solution. Although this solution exists for supercooled fluids (such that $\beta \rightarrow 1^{+}$) it can be highly inaccurate. In our final set of results we compared solutions for $\beta=1.1$. The Neumann solution has $s_{t}(0)=\infty$, with linear supercooling $s_{t}(0)=1$ and with the nonlinear relation $s_{t}(0)=e^{-Q /(P-1)}$, which for the current study on copper gave $s_{t}(0) \approx 0.278$. For large times the linear and nonlinear velocities converged (although the initial discrepancy may lead to a large difference in the position of the front) whilst the Neumann solution had $s_{t}$ approximately $50 \%$ higher even at $t=100$. Increasing the value of $\beta$ caused the three solution sets to converge. Perhaps the main conclusion of this study is that for practical purposes when attempting to predict realistic solidification rates for $\beta<1$ and even for values slightly greater than unity the nonlinear relation should be employed. Even though the Neumann solution exists it should not be trusted to predict solidification rates of supercooled liquids for values of $\beta$ close to unity.

\section{Acknowledgments}

The research of TGM was supported by a Marie Curie International Reintegration Grant Industrial applications of moving boundary problems Grant no. FP7-256417 and Ministerio de Ciencia e Innovación Grant MTM2011-23789. FF acknowledges the support of a Centre de Recerca Matemàtica PhD grant. SLM acknowledges the support of the Mathematics Applications Consortium for Science and Industry (MACSI, www.macsi.ul.ie) funded by the Science Foundation Ireland Mathematics Initiative Grant 06/MI/005.

\section{References}

[1] Mark Telford. The case for bulk metallic glass. Materials Today, 7(3): 36-43, 2004.

[2] Jan Schroers and William L. Johnson. Ductile bulk metallic glass. Phys. Rev. Lett., 93(255506), 2004. 
[3] et al Marios D. Demetriou. A damage-tolerant glass. Nature Materials, 10:123-128, 2011.

[4] Bing Li and Da-Wen Sun. Novel methods for rapid freezing and thawing of foods a review. Journal of food engineering, 54: 175-182, 2002.

[5] M. F. Ashby and D. R. H. Jones. Engineering materials 2: an introduction to microstructures, processing and design. Butterworth-Heinemann, 3rd edition, 2006.

[6] S. H. Davis. Theory of solidification. Cambridge University Press, 1st edition, 2001.

[7] Ch. Charach and B. Zaltzman. Analytic model for planar growth of a solid germ from an undercooled melt. Phys. Rev. E., 49(5): 4322-4327, 1994.

[8] J. N. Dewynne, S. D. Howison, J. R. Ockendon, and W. Xie. Asymptotic behaviour of solutions to the Stefan problem with a kinetic condition at the free boundary. $J$. Austral. Math. Soc. Ser. B, 31: 81-96, 1989.

[9] J. D. Evans and J. R. King. Asymptotic results for the Stefan problem with kinetic undercooling. Q.Jl Mech. App. Math., 53: 449-473, 2000.

[10] H. Löwen, J. Bechhoefer, and L. S. Tuckerman. Crystal growth at long times: Critical behaviour at the crossover from diffusion to kinetics-limited regimes. Phys. Rev. A, 45(4): 2399-2415, 1992.

[11] M. Merder. Solidification fronts with unusual long-time behaviour. Phys. Rev. A, 45(4): 2158-2160, 1992.

[12] T. G. Myers, S. L. Mitchell, and F. Font. Energy conservation in the one-phase supercooled Stefan problem. Int. Commun. Heat and Mass Transf., 2012. doi: http://dx.doi.org/10.1016/j.icheatmasstransfer.2012.09.005.

[13] C. Cagran, B. Wilthan, and G. Pottlacher. Enthalpy, heat of fusion and specific electrical resistivity of pure silver, pure copper and the binary $\mathrm{Ag}-28 \mathrm{Cu}$ alloy. Thermochimica Acta, 445(2): 104-110, 2006.

[14] U. Düring, J. H. Bilgram, and W. Känzing. Properties of the solid-liquid interface of growing salol crystals: A dynamic light scattering investigation. Phys. Rev. A, 30(2): 946-959, 1984.

[15] J. Mei and J. W. Davenport. Molecular-dynamics study of self-diffusion in liquid transition metals. Phys. Rev. B, 42(15): 9682-9684, 1990.

[16] I. E. Chang. Navier-stokes solutions at large distances from a finite body. J. Math. Mech., 10: 811-876, 1961.

[17] P. A. Lagerstrom and R. G. Casten. Basic concepts underlying singular perturbation techniques. SIAM Review, 14(1): 63-120, 1972.

[18] T. R. Goodman. The Heat-Balance Integral and Its Application to Problems Involving a Change of Phase. Trans. ASME, 80: 335-342, 1958.

[19] S. L. Mitchell and T. G. Myers. Application of standard and refined heat balance integral methods to one-dimensional Stefan problems. SIAM Review, 52(1): 57-86, 2010 .

[20] S. L. Mitchell. Applying the combined integral method to one-dimensional ablation. Appl. Math. Modelling, 36: 127-138, 2012.

[21] S. L. Mitchell and T. G. Myers. Improving the accuracy of heat balance integral methods applied to thermal problems with time dependent boundary conditions. Int. J. Heat Mass Trans., 53: 3540-3551, 2010.

[22] T. G. Myers. Optimizing the exponent in the heat balance and refined integral methods. Int. Commun. Heat Mass Trans., 36: 143-147, 2009.

[23] T. G. Myers. Optimal exponent heat balance and refined integral methods applied to Stefan problems. Int. J. Heat Mass Trans., 53: 1119-1127, 2010. 
[24] T. G. Myers and S. L. Mitchell. Application of the combined integral method to Stefan problems. Appl. Math. Modelling, 35: 4281-4294, 2011.

[25] S. L. Mitchell and M. Vynnycky. Finite-difference methods with increased accuracy and correct initialization for one-dimensional Stefan problems. App. Math. Comp., 215: 1609-1621, 2009.

[26] S. L. Mitchell, M. Vynnycky, I. G. Gusev, and S. S. Sazhin. An accurate numerical solution for the transient heating of an evaporating droplet. App. Math. Comp., 217: 9219-9233, 2011. 